\title{
Manejo del cólico renal en urgencias de un hospital de tercer nivel
}

\section{Nephritic colic management in an emergency room of a referral hospital}

\author{
M.A. Aibar-Arregui ${ }^{1}$, M. Matía-Sanz ${ }^{1}$, R. Pelay-Cacho', M.J. Igúzquiza-Pellejero' ${ }^{1}$, M.P. \\ Martín-Fortea $^{1}$, L. Clavel-Conget ${ }^{1}$, C. Gómez-Del Valle ${ }^{1}$, F.J. Ruiz-Ruiz ${ }^{2}$, B. Sierra-Bergua ${ }^{2}$
}

\section{RESUMEN}

Fundamento. El objetivo del presente trabajo es conocer las características epidemiológicas, el manejo diagnóstico y terapéutico, su seguimiento y la incidencia de diagnósticos alternativos en una muestra de pacientes diagnosticados de cólico renal en el Servicio de Urgencias de un Hospital de tercer nivel.

Material y métodos. Estudio descriptivo retrospectivo de 182 pacientes seleccionados aleatoriamente que consultan por clínica compatible con cólico renal en un hospital de tercer nivel, valorando su manejo inicial, seguimiento al alta y diagnósticos alternativos.

Resultados. El 55,4\% fueron varones, la edad media fue de 47,7 años y el $40 \%$ de los casos se produjeron en primavera. En todos los pacientes se realizó analítica urinaria (62,7\% tira reactiva y $72 \%$ sedimento) apareciendo alteraciones en más del $70 \%$. La función renal se deterioró en el $26,4 \%$ de los casos, siempre de forma transitoria. La prueba de imagen realizada con más frecuencia fue la radiografía de abdomen $(81,9 \%)$ seguida de la ecografía $(25,8 \%)$. El tratamiento incluyó fluidoterapia en el 31,3\% y el analgésico más usado fue el metamizol (61\%) seguido del ketorolaco (44,5\%). El 46,2\% de los pacientes necesitó más de un analgésico. Un total de 24 pacientes precisaron ingreso hospitalario y 5 de ellos cirugía urgente. El $24,1 \%$ presentó recaídas en los seis meses posteriores. El 41,6\% fue remitido al Servicio de Urología al alta. El $18,1 \%$ presentaron diagnósticos alternativos, siendo la pielonefritis aguda el más frecuente de ellos (55\%).

Conclusiones. Hemos detectado una importante variación en el manejo diagnóstico y terapéutico de estos pacientes. El uso de guías clínicas debe permitirnos unificar el manejo del paciente con cólico renal tanto en urgencias como posteriormente. El alto porcentaje de diagnósticos alternativos nos obliga a descartar sistemáticamente patologías más graves.

Palabras clave. Cólico renal. Urgencias. Diagnóstico. Tratamiento. Seguimiento. Diagnóstico diferencial.

\begin{abstract}
Background. To evaluate the diagnostic and therapeutic management of patients with nephritic colic in a referral hospital, their monitoring and the incidence of alternative diagnoses.
\end{abstract}

Methods. This is a retrospective review of 182 randomly selected patients who presented a clinical diagnosis compatible with nephritic colic in a referral hospital. In these cases initial treatment, monitoring and alternative diagnoses have been evaluated.

Results. Fifty-five point four percent of the patients were male, the mean age was 47.7 years and $40 \%$ of the cases were in spring. Urinalysis was carried out in every patient $(62.7 \%$ dipstick and $72 \%$ urinary sediment); they were pathological in over $70 \%$. In $26.4 \%$ of cases renal function deteriorated always transiently. Abdominal radiography $(81.9 \%)$ was the most frequently diagnostic test performed, followed by ultrasound $(25.8 \%)$. Treatment included a serum therapy in $31.3 \%$; metamizol (61\%) was the most commonly used analgesic followed by ketorolac (44.5\%). More than one non-steroidal anti-inflammatory was used by $46.2 \%$. Hospital admission was required by 24 patients, and 5 of them needed emergency surgery. Twenty-four point one percent of patients had relapses during the next six months. Forty-one point six percent were referred to urology discharge on from the emergency room. Eighteen point one percent of patients had alternative diagnoses; acute pyelonephritis was the most frequent of these (55\%).

Conclusions. In our work we found a significant variation in the diagnostic and therapeutic management of these patients. The use of clinical guidelines could help us to unify the management of patients with nephritic colic, both in the emergency room and on discharge. Due to the high prevalence of alternative diagnoses, we have to systematically exclude more serious diseases.

Key words. Nephritic colic. Emergency room. Diagnosis. Treatment. Monitoring. Differential diagnosis.

\section{Servicio de Medicina Interna. Hospital Clínico} Universitario. Zaragoza.

2. Servicio de Urgencias. Hospital Clínico Universitario. Zaragoza.

Recepción el 29 de enero de 2010

Aceptación provisional el 17 de febrero de 2010

Aceptación definitiva el 6 de abril de 2010

\author{
Correspondencia \\ Miguel Ángel Aibar Arregui \\ Servicio de Medicina Interna \\ Hospital Clínico Universitario \\ San Juan Bosco, 15 \\ 50009 Zaragoza \\ E-mail: Miguel_a_a_a@hotmail.com
}




\section{INTRODUCCIÓN}

El cólico renal (CR) es uno de los motivos de consulta más frecuentes en los servicios de urgencias. Aproximadamente el $12 \%$ de la población sufrirá un CR a lo largo de su vida y el $30-40 \%$ tendrá al menos una recaída en los siguientes 5 años ${ }^{1-3}$.

Estadísticamente, su frecuencia es mayor en los meses cálidos y a primera hora de la mañana, siendo los hombres de mediana edad el grupo más afectado por la enfermedad, aunque en algunas series revisadas este dato no es constante ${ }^{1,4}$.

De los pacientes afectados por un CR, el 3-10\% tienen antecedentes familiares de urolitiasis, cifra que llega al $25 \%$ si se estudian sólo los pacientes con cólicos de repetición ${ }^{1,2}$.

A pesar de su elevada incidencia, diferentes estudios han demostrado que los medios diagnósticos y el tratamiento empleados no son uniformes, atribuyéndose a diferentes causas (disponibilidad técnica, presión asistencial o existencia de atención especializada) $)^{1,5,6}$.

Otro aspecto destacable es la ausencia de estudios epidemiológicos de ámbito nacional que evalúen la verdadera importancia global de la enfermedad así como sus variaciones geográficas en función de la dieta o la composición de las aguas ${ }^{1}$. Incluso en nuestra revisión hemos encontrado pocos trabajos que analicen el seguimiento posterior al proceso agudo o el porcentaje de pacientes que acabarán precisando maniobras invasivas que no se preveían durante su evaluación inicial ${ }^{3,7}$.

Por último, conviene recordar que la frecuencia del cuadro y su forma típica de presentación nos pueden llevar a establecer un diagnóstico y un tratamiento empírico de forma precipitada, sobre todo si el paciente ya presenta antecedentes de CR. En este sentido, no debemos olvidar que el cólico renal tiene un amplio diagnóstico diferencial y que hasta en un tercio de los pacientes se puede encontrar un diagnóstico adicional o alternativo si se realiza un despistaje sistemático ${ }^{1,4,8,9}$.
El objetivo de este trabajo es conocer las características epidemiológicas, forma de presentación del cuadro, diagnóstico, tratamiento y seguimiento posterior, así como los diagnósticos alternativos de los pacientes que acuden a un servicio de urgencias de un hospital de tercer nivel con clínica sugestiva de cólico renal.

\section{MATERIAL Y MÉTODOS}

Se trata de un estudio descriptivo, retrospectivo, para el que se seleccionaron de forma aleatoria 182 pacientes que acudieron al Servicio de Urgencias del Hospital Clínico Universitario de Zaragoza por dolor en fosa lumbar y que en la clasificación inicial fueron catalogados como cólicos renales.

Todos los pacientes fueron atendidos en primer lugar por médicos especialistas en formación de medicina interna, incluyendo en el estudio a uno de cada 5 pacientes valorados de forma consecutiva entre los años 2006 y 2008.

Se recogieron datos de filiación, antecedentes personales, forma de presentación del cuadro, datos analíticos y pruebas realizadas, manejo terapéutico y seguimiento posterior incluyendo recaídas y diagnósticos alternativos al establecido inicialmente.

Los datos obtenidos fueron incluidos en una base de datos diseñada a tal efecto con el programa Microsoft Acces ${ }^{\circledR}$. El análisis-estadístico se realizó con el programa SPSS $15.0^{\circledR}$.

\section{RESULTADOS}

De los 182 pacientes incluidos en el estudio $101,(55,4 \%)$ eran varones y $81(44,5 \%)$ mujeres, con una edad media de $47,7 \pm 17,7$ años (rango 15-93). Por sexos, los hombres tenían una edad media de $48,02 \pm 15,1$ y las mujeres de $47,4 \pm 20,6$.

La época del año con mayor incidencia fue la primavera, donde se concentraron más del $40 \%$ de los casos aunque las diferencias no fueron estadísticamente significativas $(\mathrm{p}=0,630)$ (Fig. 1). 


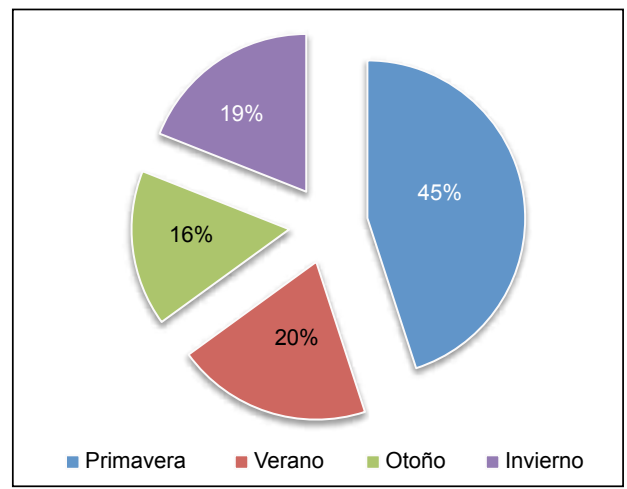

Figura 1. Porcentaje de distribución de los casos por estaciones.

En el 33,5\% de los casos constaba el antecedente personal de cólico renal, siendo este porcentaje ligeramente mayor en mujeres que en hombres (37\% frente a 30,7\%). En un paciente constaba el dato sobre antecedentes familiares de CR en la hoja de atención en urgencias.

El factor predisponerte más frecuente para padecer un CR fue la toma de diuréticos, que aparecía en 21 de los casos. En 20 de ellos se identificó el uso de diuréticos de asa (furosemida). El siguiente factor de riesgo identificado fue la patología digestiva, siendo la más frecuente la enfermedad inflamatoria intestinal. (Fig. 2).

Un total de 129 pacientes (75 varones y 54 mujeres) se presentaron en urgencias con un dolor de características típicas mientras que el $29,1 \%$ restante (53) presentaban un dolor atípico, a pesar de lo cual en la clasificación inicial fueron catalogados de CR. La duración del dolor antes de acudir a urgencias fue muy variable oscilando entre unos minutos y 10 días, con una mediana de 12 horas. No hubo diferencias en cuanto al lado afectado aunque destaca que 12 pacientes referían dolor bilateral y otros 18 dolor abdominal en el trayecto ureteral. $\mathrm{La}$ intensidad del dolor fue estratificada como leve, moderada o intensa, objetivando que el $91 \%$ de los hombres y el $95,1 \%$ de las mujeres calificaban su dolor como moderado o intenso. La puño-percusión renal fue positiva en el $68,1 \%$ de los casos.

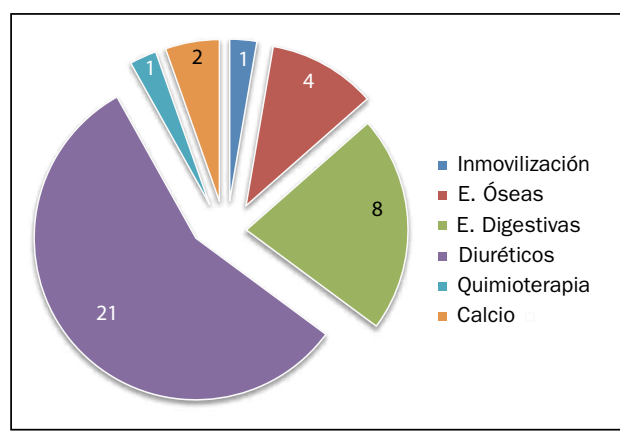

Figura 2. Factores predisponentes para padecer un cólico renal identificados en nuestros pacientes.

En cuanto a la clínica acompañante lo más frecuente fueron las náuseas $(40,1 \%)$ y la disuria $(35,7 \%)$. En la tabla 1 pueden consultarse otras manifestaciones clínicas y su distribución por sexos.

Tabla 1. Manifestaciones clínicas acompañantes.

\begin{tabular}{lrrrc}
\hline & $\begin{array}{c}\text { Total (n=182) } \\
\mathbf{N}(\%)\end{array}$ & $\begin{array}{c}\text { Varones (n=101) } \\
\mathbf{N}(\mathbf{\%})\end{array}$ & $\begin{array}{c}\text { Mujeres (n=81) } \\
\mathbf{N}(\%)\end{array}$ & p \\
\hline Sucusión + & $103(56,59)$ & $56(55,44)$ & $47(58,02)$ & 0,460 \\
\hline Náuseas & $73(40,1)$ & $43(42,6)$ & $30(37,0)$ & 0,449 \\
\hline Vómitos & $42(23,1)$ & $27(26,7)$ & $15(18,5)$ & 0,191 \\
\hline R.A.0 & $2(1,1)$ & $0(0)$ & $2(2,5)$ & 0,112 \\
\hline Disuria & $65(35,7)$ & $30(29,7)$ & $35(43,2)$ & 0,059 \\
\hline Fiebre & $8(4,4)$ & $2(2)$ & $6(7,4)$ & 0,142 \\
\hline Cefalea & $2(1,1)$ & $0(0)$ & $2(2,5)$ & 0,112 \\
\hline Hematuria & $16(8,8)$ & $9(8,9)$ & $7(8,6)$ & 0,949 \\
\hline E. C. & $4(2,2)$ & $3(3)$ & $1(1,2)$ & 0,427 \\
\hline R. & & & & \\
\hline
\end{tabular}

R.A.O: retención aguda de orina. E. C: expulsión de cálculos con la micción. 
La presencia de hematuria macroscópica fue inconstante; 16 pacientes referían haberla observado antes de su admisión en urgencias y 4 de ellos habían observado además la expulsión de "arenillas» durante la micción.

Durante su atención en urgencias se realizó una tira de orina al $62,7 \%$ de los pacientes, resultando patológica en el $70,1 \%$ de los casos (66\% de las mujeres y $73,4 \%$ de los hombres). Además se solicitó un sedimento de orina en el $72 \%$ de los casos siendo patológico en el $75,3 \%$ de ellos (66,6\% de las mujeres y $81,8 \%$ de los hombres). En la totalidad de los casos se había realizado alguna de las dos pruebas.

Se realizó extracción de sangre para hematimetría y bioquímica en 171 pacien- tes (Tabla 2), donde destacó que un 26,4\% de los pacientes presentaban un empeoramiento de la función renal respecto a sus valores previos durante el episodio agudo.

Se realizó radiografía de abdomen en 162 pacientes $(81,9 \%)$ identificando imágenes sugestivas de litiasis en $26(16,04 \%)$ de ellas. A 47 pacientes (25,8\%) se les hizo una ecografía abdominal objetivando ectasia renal en 24 de ellos (51,06\%). A lo largo del seguimiento se realizó TAC abdominal a 1 paciente y urografía intravenosa (UIV) a $37(20,3 \%)$. Tanto las solicitudes de las diferentes pruebas de imagen como el porcentaje de ecografías que demostraron ectasia fueron más frecuentes en varones (Tabla 2).

Tabla 2. Pruebas complementarias.

\begin{tabular}{|c|c|c|c|c|}
\hline & Total & Hombres & Mujeres & \multirow{2}{*}{$\mathbf{p}$} \\
\hline Sedimento & $\mathrm{N}(\%)$ & $\mathrm{N}(\%)$ & $\mathrm{N}(\%)$ & \\
\hline Patológico & $116(63,7)$ & $72(71,3)$ & $44(54,3)$ & \multirow{3}{*}{0,096} \\
\hline Normal & $38(20,9)$ & $16(15,8)$ & $22(27,2)$ & \\
\hline $\mathrm{NR} / \mathrm{NC}$ & $28(15,3)$ & $13(12,8)$ & $15(18,5)$ & \\
\hline Tira orina & $\mathrm{N}(\%)$ & $\mathrm{N}(\%)$ & $\mathrm{N}(\%)$ & $\mathbf{p}$ \\
\hline Patológica & $80(44,0)$ & $47(46,5)$ & $33(40,7)$ & \multirow{3}{*}{0,723} \\
\hline Normal & $34(18,7)$ & $17(16,8)$ & $17(21,0)$ & \\
\hline $\mathrm{NR} / \mathrm{NC}$ & $68(37,3)$ & $37(36,6)$ & $31(38,2)$ & \\
\hline Hemograma & N (\%) & $\mathrm{N}(\%)$ & $\mathrm{N}(\%)$ & $\mathbf{p}$ \\
\hline Normal & $100(54,9)$ & $50(49,5)$ & $50(61,7)$ & \multirow{4}{*}{0,228} \\
\hline Leucocitosis $+\mathrm{N}$ & $46(25,3)$ & $29(28,7)$ & $17(21,0)$ & \\
\hline Leucocitosis - N & $25(13,7)$ & $17(16,8)$ & $8(9,9)$ & \\
\hline $\mathrm{NC} / \mathrm{NR}$ & $11(6,0)$ & $5(5,0)$ & $6(7,4)$ & \\
\hline I. renal & $48(26,4)$ & $40(39,6)$ & $8(9,9)$ & 0,000 \\
\hline Rx abdomen & $162(89,0)$ & $94(93,1)$ & $68(84,0)$ & 0,060 \\
\hline Litiasis Rx & $26(16,0)$ & $19(20,2)$ & $7(10,3)$ & 0,088 \\
\hline Ecografía & $47(25,8)$ & $28(27,7)$ & $19(23,5)$ & 0,513 \\
\hline Ectasia & $24(51,0)$ & $15(53,5)$ & $9(47,3)$ & 0,577 \\
\hline U.I.V & $37(20,3)$ & $23(22,8)$ & $14(17,3)$ & 0,361 \\
\hline T.A.C & $1(0,5)$ & $0(0)$ & $1(1,2)$ & 0,263 \\
\hline
\end{tabular}

NR/NC: no realizado o no consta. $\pm \mathrm{N}$ : con o sin neutrofília.

UIV: urografía intravenosa. TAC: tomografía axial computarizada.

I. Renal: insuficiencia renal. 
El tratamiento instaurado en urgencias incluyó fluidoterapia endovenosa en el $31,3 \%$ de los casos. Los fármacos más usados fueron los antiinflamatorios no esteroideos (AINE) prescritos en el 81,3\% de los casos, seguidos de los procinéticos (metoclopramida) y los inhibidores de la bomba de protones (Tabla 3). Un total de
111 pacientes recibieron al menos una dosis de metamizol durante su estancia en el servicio de urgencias. Más de un tercio de los enfermos precisaron al menos de dos analgésicos para el control del dolor $(47,8 \%)$. El fármaco más prescrito al alta de urgencias fue el metamizol $(59,9 \%)$ seguido del diclofenaco $(23,6 \%)$.

Tabla 3. Fármacos utilizados en urgencias.

\begin{tabular}{lrrrr}
\hline & $\begin{array}{c}\text { Total } \\
\mathbf{N}(\%)\end{array}$ & $\begin{array}{c}\text { Varones } \\
\mathbf{N}(\%)\end{array}$ & $\begin{array}{c}\text { Mujeres } \\
\mathbf{N}(\%)\end{array}$ & p \\
\hline AINE & $148(81,3)$ & $84(83,2)$ & $64(79,0)$ & 0,497 \\
\hline Diclofenaco & $17(9,3)$ & $11(10,9)$ & $6(7,4)$ & 0,214 \\
\hline Ketorolaco & $81(44,5)$ & $50(49,5)$ & $31(38,3)$ & 0,111 \\
\hline Ibuprofeno & $5(2,7)$ & $4(4,0)$ & $1(1,2)$ & 0,156 \\
\hline \multicolumn{1}{c}{ Metamizol } & $111(61,0)$ & $63(62,4)$ & $48(59,3)$ & 0,277 \\
\hline Paracetamol & $27(14,8)$ & $15(14,9)$ & $12(14,8)$ & 0,283 \\
\hline Opioides & $22(12,0)$ & $11(10,9)$ & $11(13,5)$ & 0,112 \\
\hline \multicolumn{1}{c}{ Tramadol } & $17(9,3)$ & $9(8,9)$ & $8(9,9)$ & 0,273 \\
\hline Petidina & $3(1,6)$ & $0(0)$ & $3(3,7)$ & $\mathbf{0 , 0 4 1}$ \\
\hline \multicolumn{1}{c}{ Cloruro mórfico } & $2(1,1)$ & $2(2,0)$ & $0(0)$ & 0,112 \\
\hline Corticoides & $24(13,2)$ & $18(17,8)$ & $6(7,4)$ & $\mathbf{0 , 0 3 8}$ \\
\hline Buscapina & $8(4,4)$ & $5(5,0)$ & $1(1,2)$ & 0,155 \\
\hline Metoclopramida & $61(33,5)$ & $12(11,9)$ & $5(6,2)$ & 0,275 \\
\hline IBP & $50(27,5)$ & $27(26,7)$ & $23(28,4)$ & 0,266 \\
\hline ANE: antin & & &
\end{tabular}

AINE: antiinflamatorios no esteroideos.

IBP: inhibidores de la bomba de protones

De los 182 pacientes incluidos en el estudio, 24 (13,2\%) precisaron ingreso hospitalario y $5(2,7 \%)$ cirugía urgente. Se produjeron un total de 44 recaídas precoces (menos de 6 meses); de ellas, 37 fueron en pacientes que habían tomado AINE como único tratamiento.

Respecto al seguimiento de los pacientes, el $41,6 \%$ fue valorado en consultas externas de urología tras el episodio agudo $\mathrm{y}$, en lo que se refiere al diagnóstico definitivo, la pielonefritis aguda fue el principal diagnóstico alternativo y un total de 33 pacientes $(18,1 \%)$ presentaron patologías diferentes al CR (Fig. 3).

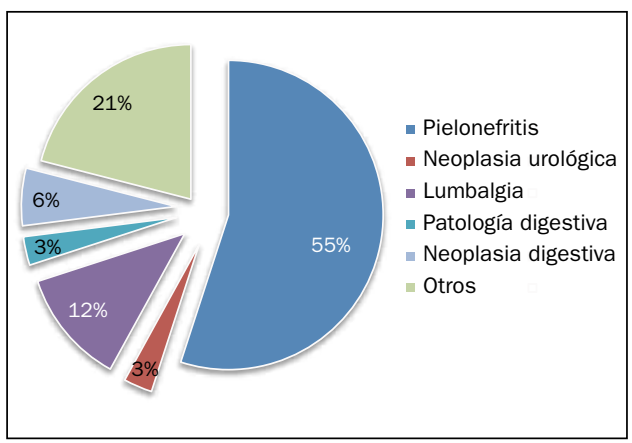

Figura 3. Diagnósticos alternativos al cólico renal encontrados en nuestros pacientes, expresados en porcentaje. 


\section{DISCUSIÓN}

El cólico renal es una de las urgencias urológicas más frecuentes y requiere un diagnóstico y tratamiento rápido debido al dolor intenso que produce y al deterioro de la función renal a que conduce. Además, es uno de los motivos más frecuentes de consulta en el Servicio de Urgencias por su elevada incidencia en la población general y la alta tasa de recidivas ${ }^{1}$.

Este trabajo pretende analizar las características epidemiológicas y clínicas así como el manejo en urgencias y el seguimiento posterior de una muestra aleatoria de pacientes que consultan en urgencias por un dolor sugestivo de CR, con especial atención al diagnóstico definitivo una vez concluido el estudio del paciente.

Existen pocos trabajos epidemiológicos de ámbito nacional o internacional. Además, llama la atención la relativa escasez de guías clínicas útiles y el diferente manejo del cuadro agudo según los autores consultados, algunos de los cuales enfatizan la necesidad de contar con guías específicas sobre el manejo en urgencias y el seguimiento posterior del enfermo ${ }^{1-3,10}$.

La mayor parte de los CR son causados por una obstrucción ureteral aguda por un cálculo (90\%), aunque también puede deberse a alteraciones no litiásicas de la vía urinaria o incluso a compresiones extrínsecas del uréter por procesos expansivos o inflamatorios intraabdominales. En todos los casos, la oclusión completa o incompleta de las vías urinarias provoca una distensión aguda del sistema colector que es el origen del dolor, quedando la irritación de la mucosa por el cálculo en un segundo lugar ${ }^{1}$.

Clásicamente se ha descrito una incidencia mayor en hombres que en mujeres, 3:1 según algunos autores ${ }^{11}$; en nuestra serie esta proporción no se mantiene aunque sigue siendo mayor el número de varones. La estación del año en la que se recogieron más casos fue la primavera, aunque hay pequeñas diferencias si se analizan por separado hombres y mujeres (Tablas 1-3); el aumento de incidencia de CR durante los meses cálidos es habitual y, según estudios recientes, afectaría más a varones de mediana edad, lo que podría justificar las diferencias encontradas en nuestra serie ${ }^{12}$.

El antecedente de cólico renal constaba en más de un tercio de nuestros pacientes, cifra superior a la que han reportado otras revisiones ${ }^{1}$. En este sentido, queremos destacar que ya en anteriores trabajos de nuestro grupo se reportó una mayor incidencia de litiasis renal en nuestra área sanitaria con respecto a otros estudios locales en diferentes zonas del país; sin duda y, en ausencia de un estudio epidemiológico más amplio, estos datos deben interpretarse con cautela ya que proceden de muestras reducidas de pacientes. Una posible explicación la aportan Millán y col en su reciente revisión sobre la composición de las aguas en diferentes capitales españolas y que coincide con nuestros trabajos al señalar la importancia del alto contenido en calcio de las aguas de nuestra ciudad ${ }^{13,14}$. En este mismo sentido, nos parece destacable el hecho de que casi el $12 \%$ de los pacientes incluidos en nuestro estudio estaban tomando diuréticos de asa (furosemida) en el momento del cuadro agudo ya que dicho fármaco también se ha relacionado con la formación da cálculos de oxalato cálcico y ésta es la composición más frecuente en nuestro medio ${ }^{1,13}$.

El dolor típico del cólico renal es de inicio brusco, unilateral y muy intenso, se localiza en fosa lumbar y se irradia característicamente por el trayecto ureteral hasta genitales externos e incluso a la cara interna del muslo; es típica así mismo la gran agitación del paciente que no puede encontrar una postura antiálgica ${ }^{1}$. A pesar de que estas características están ampliamente descritas y reconocidas, hasta un $25 \%$ de los pacientes se presentan con dolores atípicos referidos a abdomen o en forma de espasmos o calambres; en nuestra serie casi un $30 \%$ fueron dolores atípicos, un porcentaje mayor al descrito en otros estudios como el de Browm o el de Serinken $\mathrm{y} \mathrm{col}^{10,15}$.

Debido a la gran intensidad del dolor la mayoría de los pacientes, en particular 
aquellos que presentan recaídas, no tardan en iniciar el tratamiento por su cuenta o en acudir a urgencias. En nuestra serie más del 90\% de ellos refieren el dolor como moderado o intenso y la práctica totalidad acuden en las primeras 2-3 horas desde el inicio; aun así, hemos recogido algunos pacientes que tardaron hasta 10 días en consultar con un médico y uno de ellos hubo de ser intervenido de forma urgente por la formación de una gran colección perirrenal.

Además del dolor, la disuria y las náuseas son los síntomas más habituales que acompañan al CR. La disuria, producida por irritación vesical, fue más frecuente en mujeres, mientras que las náuseas y los vómitos lo fueron en los varones; ambas manifestaciones clínicas fueron más frecuentes en nuestra serie que en las revisadas para la elaboración de este trabajo, sin que hayamos podido encontrar una explicación clara para ello, aunque el hecho de que todos nuestros pacientes fueran recogidos en un hospital y tras un triaje previo podría explicar en parte la mayor intensidad de los síntomas con respecto a otras series que incluyen pacientes con manifestaciones clínicas menos intensas. Esto tendría relación con el hecho de que en nuestra serie el porcentaje de pacientes que habían presentado hematuria de esfuerzo o expulsión de cálculos con la orina fuera mayor que en dichos trabajos ${ }^{10,15}$.

El diagnóstico inicial del cólico renal no complicado debe basarse en una cuidadosa historia clínica y exploración física y, en la mayor parte de los casos, una tira reactiva de orina o un sedimento urinario patológicos son suficientes para establecer un diagnóstico ${ }^{1}$. Sin embargo, en nuestra revisión hemos encontrado una gran variación en las pruebas solicitadas tanto en el servicio de urgencias como en el seguimiento posterior de los pacientes; por ejemplo, con respecto a algunas series en las que el porcentaje de pacientes a los que se había solicitado un estudio de la orina no llegaba al $75 \%$, en nuestra serie y otras de nuestro entorno se he solicitado al $100 \%$ de los enfermos ${ }^{4,15}$.
El análisis de sangre no es imprescindible en la evaluación inicial de un cólico renal no complicado y su principal indicación es la sospecha de complicaciones (fiebre, oligoanuria, obstrucción bilateral, monorrenos, etc.) valorando fundamentalmente la leucocitosis con o sin neutrofília y el deterioro de la función renal ${ }^{1}$.

En casi el 94\% de nuestros enfermos se solicitó un análisis de sangre donde lo más destacable es el alto porcentaje de pacientes que mostraban empeoramiento de la función renal respecto a valores previos, si bien en todos los casos fue un empeoramiento transitorio que mejoró tras el tratamiento médico o instrumental; no hemos documentado ningún caso de deterioro irreversible de la función renal.

El papel de la radiografía de abdomen es muy limitado tanto por su baja sensibilidad como por el hecho de que no va a cambiar nuestra actitud terapéutica prácticamente nunca. Algunos estudios cifran la sensibilidad de la radiografía entre el $45-60 \%$ de los casos para detectar cálculos o signos indirectos de litiasis renal e incluso se ha tratado de estudiar si esta sensibilidad depende de la experiencia del observador sin encontrar resultados concluyentes ${ }^{16-18}$.

A pesar de estas evidencias, en un gran porcentaje de nuestros pacientes (89\%) se incluye una radiografía en la evaluación inicial y, en este sentido, queremos destacar que tan sólo en un $16 \%$ de los casos se detectó una imagen sugestiva de litiasis, un porcentaje muy bajo, máxime si tenemos en cuenta que más del $70 \%$ de los cálculos analizados en nuestro hospital contienen materiales radiopacos ${ }^{13}$.

En nuestra opinión, la radiografía sólo estaría indicada como exploración inicial en casos con dudas diagnósticas y si no se dispone de otras técnicas de imagen con mayor rentabilidad, ya que a pesar de sus limitaciones puede contribuir al diagnóstico diferencial con otros procesos ${ }^{19}$.

En una reciente revisión, Esquena y col resumen las principales ventajas e inconvenientes de otras pruebas de imagen utilizadas en la valoración del $\mathrm{CR}^{1}$. Para 
estos autores la ecografía es un método incruento, portátil, repetible y barato que no utiliza radiaciones y que puede contribuir al diagnóstico diferencial con otros procesos. Además, el uso del doppler puede valorar el funcionalismo renal y eventualmente sustituir a la urografía intravenosa (UIV), sobre todo si aparecen contraindicaciones para su realización. La principal limitación es que puede pasar por alto hasta el $30 \%$ de las ectasias pielo-caliciales en el momento inicial. Para algunos autores la ecografía debería ser la prueba de referencia para el cólico renal no complicado ya que, aunque la rentabilidad de la tomografía helicoidal (TAC) sin contraste sea mayor, la disponibilidad, el coste y la ausencia de radiación de la ecografía son suficientes argumentos para recomendarla de inicio ${ }^{18}$.

Como sucedía con el análisis de orina, la utilización de la ecografía es desigual según las series revisadas y, mientras en nuestro hospital se realizó en tan sólo el $25 \%$ de los pacientes, hemos encontrado trabajos en los que la ecografía fue la única prueba de imagen realizada hasta en el $70 \%$ de los $\operatorname{casos}^{20}$.

La UIV sigue siendo el método de referencia para el diagnóstico del CR agudo en algunos centros ya que es fiable, barata y relativamente inocua; sin embargo, parece que la introducción del doppler y de la TAC sin contraste hará que su uso sea cada vez menor $^{21}$.

La TAC sin contraste es en la actualidad el mejor método diagnóstico para el CR agudo, con una sensibilidad y especificidad próximas al $100 \%$ y con un coste no superior a la UIV, tan sólo la incapacidad de valorar la función renal aparece como un inconveniente. A todo esto hay que añadir la gran cantidad de información que aporta en el diagnóstico diferencial con otros procesos, ya sean responsables del cuadro o como hallazgos exploratorios ${ }^{1,2,8,9}$.

Una vez más observamos en nuestra revisión de la literatura que, a pesar de la evidencia científica en torno a su uso, la TAC ha sido solicitada de forma desigual en las diferentes series ${ }^{15,20}$. En nuestro caso, tan sólo un paciente ha sido valorado mediante esta técnica. Otros autores como Cupisti y col ya han reportado series en las que la limitación técnica o económica limita el uso de la TAC como primera aproximación diagnóstica ${ }^{20}$.

El tratamiento médico del CR debe incluir analgesia, control de los síntomas acompañantes, tratamiento expulsivo $\mathrm{y}$ prevención de recaídas.

La intensidad del dolor hace necesario en la mayoría de los casos iniciar el tratamiento analgésico antes de tener un diagnóstico definitivo. La revisión de la literatura nos indica que el tratamiento de elección son los AINE y entre ellos el diclofenaco y el ketorolaco parecen los más eficaces. Los opiáceos no han demostrado ser superiores a la hora de reducir el dolor e incluso algunos estudios sugieren que el porcentaje de recaídas es mayor cuando se utilizan como tratamiento úni$\mathrm{co}^{22-24}$.

La elección del analgésico y de la vía de administración depende en gran medida de la intensidad del dolor y del ámbito en el que nos encontramos. En nuestro medio, el $81 \%$ de los pacientes han sido tratados al menos con un AINE siendo el metamizol el más prescrito, mientras que en otros estudios el $100 \%$ de los pacientes han tomado AINEs y, en particular en Estados Unidos, el metamizol no se utiliza prácticamente nunca $^{15,20}$.

El paracetamol, utilizado en casi el 15\% de nuestros enfermos no se considera de primera línea y tan sólo estaría indicado en casos de alergia a AINE o intolerancia a los mismos; en este mismo sentido, los anticolinérgicos, usados ampliamente en el pasado y tan sólo en el 4,4\% de nuestros casos, no están indicados e incluso podrían ser contraproducentes por retrasar la expulsión del cálculo ${ }^{1}$.

Otro de los aspectos importantes en relación con la analgesia es la frecuente necesidad de utilizar más de un fármaco para el control del dolor; en nuestra serie casi la mitad de los pacientes precisaron al menos dos analgésicos en urgencias. Otros auto- 
res como Browm ya han documentado esta necesidad e incluso en su serie el porcentaje de pacientes polimedicados fue superior al de la nuestra ${ }^{15}$.

Un porcentaje no despreciable de pacientes $(12,1 \%)$ no precisó tratamiento analgésico en urgencias probablemente porque ya habían iniciado el tratamiento por su cuenta antes de acudir a dicho servicio.

Los fármacos utilizados con mayor frecuencia para el tratamiento de las manifestaciones acompañantes y la prevención de complicaciones fueron la metoclopramida $(33,5 \%)$ y los inhibidores de la bomba de protones (27,5\%); ambos fueron prescritos a domicilio de manera mucho más frecuente en varones.

El tratamiento expulsivo puede incluir calcioantagonistas, corticoides y/o alfa bloqueantes como la tamsulosina y ha demostrado mejorar el porcentaje de pacientes que expulsan de manera espontánea el cálculo; a pesar de ello, su uso no está generalizado en nuestro medio y, de hecho, ninguno de nuestros pacientes recibió este tratamiento ${ }^{16,25,26}$.

El uso de diuréticos y fluidoterapia intensa usado en el pasado no tiene validez hoy en día y se considera contraindicada la sobrehidratación durante el episodio agudo. Sin embargo, sigue siendo práctica habitual en algunos centros donde hasta el $63 \%$ de los pacientes (31\% en nuestra serie) reciben fluidoterapia intraveno$\mathbf{s a}^{4,10,27}$.

En lo que se refiere a la prevención de recaídas, la dieta y la calidad del agua de consumo son pilares fundamentales, sobre todo en los pacientes con antecedentes familiares o con historia de cólicos de repetición.

En la revisión de la literatura, apenas se encuentran estudios de seguimiento tras el alta del servicio de urgencias. En una revisión reciente de Sterret y col, el $61 \%$ de los pacientes atendidos en urgencias fueron posteriormente valorados por un urólogo en consultas. En nuestra serie, el $41,6 \%$ de los pacientes acudieron a las consultas de urología en los 6 meses posteriores a su atención en urgencias.

Por último, queremos comentar que un alto porcentaje de pacientes $(18,1 \%$ en nuestra serie y hasta un $30 \%$ en otras) que inicialmente se catalogan como CR simple presentan diagnósticos diferentes una vez concluido el estudio; la pielonefritis aguda es, en la mayoría de las series, el diagnóstico alternativo más frecuente ${ }^{8,9}$.

La existencia de guías clínicas validadas debería permitirnos unificar el proceso diagnóstico y el tratamiento de los pacientes, tanto durante la fase aguda como en el seguimiento posterior. Sin embargo, tal como se desprende del análisis de nuestros resultados, tanto las pruebas solicitadas a los pacientes como el tratamiento instaurado son variables y no siempre se ajustan a la evidencia científica actual. Un claro ejemplo es la gran cantidad de radiografías de abdomen frente a la escasez de ecografías u otras pruebas de imagen solicitadas. Hay que considerar que estas pruebas no sólo ayudan al diagnóstico sino que contribuyen a descartar otros procesos.

En nuestro trabajo no hemos detectado ningún dato que identifique a los pacientes con mayor frecuencia de diagnósticos alternativos o adicionales. En nuestra opinión, el alto porcentaje de pacientes que los presentan nos debe obligar al despistaje sistemático de patologías potencialmente más graves, en particular en los pacientes con evolución tórpida o recaídas frecuentes que sí podrían ser un subgrupo de mayor riesgo.

En conclusión, el uso de guías clínicas debe permitirnos unificar el manejo del paciente con cólico renal tanto en urgencias como posteriormente.

El alto porcentaje de diagnósticos alternativos nos obliga a descartar sistemáticamente patologías más graves. Para ello, la prueba más rentable es la TAC, aunque su menor coste, la ausencia de contraindicaciones y la mayor disponibilidad hacen que la ecografía sea el método de imagen más recomendado. 


\section{BIBLIOGRAFÍA}

1. Esquena S, Millán F, Sánchez FM, Rousaud F, Marchant F, Villavicencio H. Cólico renal: revisión de la literatura y evidencia científica. Actas Urol Esp 2006; 30: 268-280.

2. TEICHMAn J. Acute renal colic from ureteral calculus. N Engl J Med 2004; 350: 684-693.

3. Sterrett S, Moore N, Nakada S. Emergency room follow up trends in urolithiasis: single center report. Urology 2009; 73: 1195-1198.

4. Аmat M, Romero P. Cólico nefrítico. Análisis de 140 casos. Actas Urol Esp 1994; 18: 855860 .

5. Preminger GM, Tiselius HG, Assimos DG, Alken P, Buck C, Galluci M et al. Guideline for the management of ureteral calculi. J Urol 2007; 178 : 2418-2434.

6. Lasoye T, Sedgwick PM, Patel N, Skinner C, NAYEEM N. Management of acute renal colic in the UK: a questionnaire survey. BMC Emerg Med 2004; 4: 5.

7. Papa L, Stiell iG, Wells GA, Ball I, Battram E, MAHONEY JE. Predicting intervention in renal colic patients after emergency department evaluation. Can J Emerg Med 2005; 7: 78-86.

8. Ahmad NA, HAMmad M, ReEs J. Incidental diagnosis of diseases on un-enhanced helical computed tomography performed for ureteric colic. BMJ Urology 2003; 3: 2. [Disponible en www.biomedcentral.com/1471-2490/3/2].

9. Rucker CM, Menias CO, Bhalla S. Mimics of renal colic: alternative diagnoses at unhenhanced helical CT. Radiographics 2004; 24 : S11-S33.

10. Serinken M, Karcioglu O, Turkcuer I, Ozkan Hi, Kemal M, Bukiran A. Analysis of clinical and demographic characteristics of patients presenting with renal colic in the emergency department. BMC Res Notes 2008; 1; 79.

11. DurÁn R, HERnández C. Litiasis urinaria. Medicine 2003; 8: 6055-6065.

12. Chauhan V, Eskin B, Allegra JR, Cochrane DG. Effect of season, age, and gender on renal colic incidence. Am J Emerg Med 2004; 22: 560-563.

13. Aibar MA, Gutiérrez AP, Rodrigo MP, Laborda K, HeRnÁNDEZ AB, Blasco G. Litiasis renal en el área III de Zaragoza: bioquímica y epidemiología. Actas Urol Esp 2004; 28: 661-665.

14. Millán F, Gracia S, Jiménez R, Serrano M, Rousaud F, SÁnchez F et al. Análisis de las aguas embotelladas y de grifo españolas y de las implicaciones de su consumo en la litiasis urinaria. Actas Urol Esp 2009; 33: 778-793.

15. Brown J. Diagnostic and treatment patterns for renal colic in US emergency departments. Intern Urol Nephrol 2006; 38: 87-92.

16. Masarani M, DinneEn M. Ureteric colic: new trends in diagnosis and treatment. Postgrad Med J 2007; 83: 469-472.

17. Merenciano FJ, Escuder A, Manzanero MA, MarTíNEZ P, RAFIE W, AMAT M et al. El valor de la radiología simple de abdomen en el cólico nefrítico. Actas Urol Esp 2000; 24: 138-143.

18. Del arco C, Parra ML, García-Casasola G. Pruebas de imagen en el dolor abdominal agudo. Rev Clin Esp 2008; 208: 520-524.

19. Romero P, Martínez MC Cólico nefrítico, teratoma ovárico y radiografía simple del aparato urinario. Actas Urol Esp 2007; 31: 936-937.

20. Cupisti A, Pasquali E, Lusso S, Carlino F, Orsitto E, Melandri R. Renal colic in Pisa emergency department: Epidemiology, diagnostics and treatmente patterns. Intern Emerg Med 2008; 3: 241-244.

21. Shokeir AA, Abdoulmaaboud M. Prospective comparison of nonenhanced helical computerized tomography and Doppler ultrasonography for the diagnosis of renal colic. J Urol 2001; 165: 1082-1084.

22. Holdgate A, Pollock T. Nonsteroidal anti-inflammatory drugs (NSAIDs) versus opioids for acute renal colic. Cochrane Database Syst Rev 2005; 18: CD004137.

23. Holdgate A, Pollock T. Systematic review of the relative efficacy of nonsteroidal antiinflammatory drugs and opioids in the treatment of acute renal colic. BMJ 2004; 328; 1401-1404.

24. Labrecque M, Dostaler LP, Rousselle R, Nguyen T, POIRIER S. Efficacy of non-steroidal anti-inflammatory drugs in the treatment of acute renal colic. A meta analysis. Arch Intern Med 1994; 154: 1381-1387.

25. Sterret S, NAKADA S. Medical expulsive therapy. Curr Opin Urol 2008; 18: 210-213.

26. Hollingsworth JM, Rogers MAM, Kaufman S, BRADFORD TJ, SAINT S, Wei JT et al. Medical therapy to facilitate urinary stones passage: a meta analysis. Lancet 2006; 368: 1171-1179.

27. RozAnski TA, EDMONSON JM. Fluids and diuretics for acute ureteric colic. Cochrane Database Syst Rev 2005; 20 (3): CD004926. 